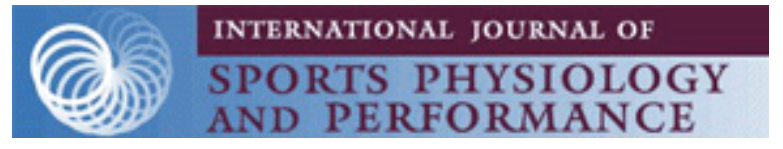

\title{
Relationship between tethered swimming in a flume and swimming performance
}

\begin{tabular}{|r|l|}
\hline Journal: & International Journal of Sports Physiology and Performance \\
\hline Manuscript ID & IJSPP.2019-0466.R2 \\
\hline Manuscript Type: & Original Investigation \\
\hline Date Submitted by the & 22-Oct-2019 \\
\hline Complete List of Authors: & $\begin{array}{l}\text { Ruíz-Navarro, Jesús J.; University of Granada, Physical Education and } \\
\text { Sports Department } \\
\text { Morouço, Pedro; Polytechnic Institute of Leiria, Department of Human } \\
\text { Kinetics; Universidade de Lisboa, Laboratory of Biomechanics and } \\
\text { Functional Morphology, Interdisciplinary Centre for the Study of Human } \\
\text { Performance (CIPER) } \\
\text { ARELANO, RAUL; University of Granada, Physical Education and Sports } \\
\text { Department; Raúl Arellano, Raúl Arellano }\end{array}$ \\
\hline Keywords: & \begin{tabular}{l} 
sport, exercise performance \\
\hline
\end{tabular} \\
\hline
\end{tabular}

\section{SCHOLARONE $^{\text {IM }}$ Manuscripts}




\begin{abstract}
Purpose: This research aimed to study the relationship between tethered swimming in a flume at different speeds and swimming performance. Methods: Sixteen regional level swimmers performed 25, 50 and 100-m front crawl trials and four 30-s tethered swimming tests at zero, 0.926, $1.124,1.389 \mathrm{~m} \cdot \mathrm{s}^{-1}$ water flow velocities. Average and maximum force, average and maximum impulse, and intra-cyclic force variation $(\mathrm{dF})$ were estimated for each tethered swimming trial. Swimming velocity and intracyclic velocity variation $(\mathrm{dv})$ were obtained for each freeswimming trial. Stroke rate and rate of perceived effort were registered for all trials. Results: Tethered swimming variables, both at $1.124 \mathrm{~m} \cdot \mathrm{s}^{-1}$ and at $1.389 \mathrm{~m} \cdot \mathrm{s}^{-1}$ water flow velocities, were positively associated with $25-\mathrm{m}$ swimming velocity $(p<0.05)$. Average force and maximum impulse in stationary swimming were significantly associated with $25-\mathrm{m}$ swimming velocity $(p<0.05)$. A positive relationship between water flow velocities with $\mathrm{dF}$ was observed. Swimming performance was not related to $\mathrm{dF}$ or $\mathrm{dv}$. Neither stroke rate, nor rate of perceived exertion differed between the 4 tethered conditions and mean $50-\mathrm{m}$ free swimming velocity ( $p>0.05)$. Conclusions: Measuring force in a swimming flume at higher water flow velocities is a better indicator of performance than stationary tethered swimming. It allows assessing the ability to effectively apply force in the water.
\end{abstract}

Keywords: tethered forces; strength; training; exercise testing; force assessment 


\section{Introduction}

Performance in competitive swimming is measured through the time spent to complete an established distance. Muscular force production while stroking ${ }^{1}$, swimming technique ${ }^{2}$, and aerobic/ anaerobic energy production ${ }^{3}$ are determinants in competitive swimming performance. Over short distances, the force exerted in water must be high to overcome the water resistance ${ }^{4}$. For that reason, the assessment of the force exerted in swimming becomes extremely important ${ }^{5}$. However, the aquatic environment complicates the direct measurement of force application during swimming performance ${ }^{6}$. Experimental techniques such as Measurement of Active Drag, Velocity Perturbation Method or Assisted Towing Method have been used to calculate mean propulsive force. These methods calculate mean propulsive force relying on computing active drag rather than measuring the force independently ${ }^{7}$, since the main active drag force may be considered as identical in magnitude to the mean propulsive force at a constant speed.

The direct measurement of force has been obtained through tethered swimming, which has been proposed as a valid and reliable methodology to assess swimmer's strength potential ${ }^{6,8,9}$. Moreover, physiological variables in tethered swimming are not significantly different to free swimming of similar duration ${ }^{5}$. Still, there are kinematical differences between free swimming and tethered swimming ${ }^{10}$, especially in the first half of the aquatic path where the hand is oriented perpendicular earlier and velocity and acceleration differs ${ }^{11}$.

Tethered swimming is a tool to measure the exerted forces in water, assessing individual force-time curves during the exercise $^{12}$. The most common parameters obtained are: average $^{13}$ and maximum force ${ }^{1}$, average and maximum impulse ${ }^{5}$. Nevertheless, there is no clear evidence suggesting which one is the most reliable parameter; demonstrating that more studies are required to better understand this topic. Considering that propulsion occurs during the whole propulsive phase of the stroke cycle ${ }^{14}$, the relation between force and time should be considered as follows ${ }^{5}$ :

$$
I=\int_{t 1}^{t 2} F \cdot d t
$$

Where $I$ represents the impulse and $F$ is the applied force from time $t_{1}$ to $t_{2}$. Thus, calculations of the impulse of force may be more accurate when analysing the tethered forces ${ }^{15}$, as the impulse of force depends on the magnitude, duration, and direction of the applied force. In addition, measurements combining force and speed may be more accurate and related to performance ${ }^{16}$.

Recently, a new parameter related to tethered force has been proposed; intra-cyclic force variation $(\mathrm{dF})^{17}$. This variable seems to be effective in evaluating the swimmer's ability to effectively apply force in the water and is highly associated with 
performance. On the contrary, the intra-cyclic velocity variation $(\mathrm{dv})$ is one of the most applied parameters by academics and practitioners to evaluate the efficiency of swimmers, even though the relationship with performance is not completely clear yet ${ }^{18}$.

The main differences between free swimming and tethered swimming are the stationary water and the non-displacement of the swimmers. It is suggested that using a swimming flume would be a state more similar to free swimming than tethered swimming at zero velocity ${ }^{19}$; however, to our knowledge, there is insufficient evidence of previous research which studies the effects of implementing a swimming flume on tethered swimming variables and how it would affect the relationship with swimming performance over short distances.

Therefore, the scarce knowledge and limitations regarding tethered swimming demonstrate the need to know whether a closer situation to free swimming could be achieved by the employment of a flume. Thus, this research aimed to study the relationships between tethered swimming in a flume at different speeds and swimming performance. It was hypothesized that higher associations would be observed when the water flow velocity was closer to the free-swimming velocity.

\section{Methods}

\section{Subjects}

Sixteen regional male swimmers participated in the study $(19.6 \pm 3.3$ years of age, $176.1 \pm 4.5 \mathrm{~cm}$ in height, $70.7 \pm 9.5 \mathrm{~kg}$ of body mass, $58.24 \pm 2.2$-s of long course $100-\mathrm{m}$ freestyle personal best, representing $76 \pm 5 \%$ of the World record). The swimmers were required to have at least 5 years of experience in competitive swimming, as inclusion criteria. The protocol was fully explained to the participants before they provided written consent to participate. The study was conducted according to the Code of Ethics of the World Medical Association (Declaration of Helsinki), and the protocol was approved by the university ethics committee.

\section{Design}

A cross-sectional study design was used. Swimming performance was tested in a 25-m swimming pool (25-m x 16.5$\mathrm{m})$ (water temperature $=27^{\circ}$, humidity $=65 \%$ ) and tethered forces were tested in a swimming flume (Endless Pool Elite Techno Jet Swim 7,5, HP, Aston PA, USA) with predefined velocity range and with flow velocity being measured at $0.30 \mathrm{~cm}$ depth using an FP101 flow probe (Global Water, Gold River, $\mathrm{CA}^{20}$ ) (water temperature $=26^{\circ}$, humidity $=52 \%$ ). Swimmers were assessed on two consecutive days in the same conditions. 
To improve the reliability of the measurements, participants were asked to refrain from intense exercise the day prior to and on the test days. Moreover, they were asked to abstain from caffeine, alcohol or any stimulant drink during those days. Tests execution orders were randomly assigned and performed in the same conditions. Tests were preceded by a standardised warm up, which consisted of $1000-\mathrm{m}$ of low to moderate intensity front crawl swimming (400-m swim, 100-m pull, 100-m kick, 4x50$\mathrm{m}$ at increasing speed, 200-m easy swim) ${ }^{17}$.

\section{Methodology}

The tethered swimming test consisted of 30-s arm stroke (without leg action) in 4 different conditions: at zero velocity, which replicates the measurement in the pool, and at 3 different velocities of water flow: $0.926,1.124$ and $1.389 \mathrm{~m} \cdot \mathrm{s}^{-1}$. These 3 velocities were chosen after a pilot study, representing $50 \%$ of the maximum swimming velocity, the easy swimming velocity, and the maximum velocity that allow registering all the forces of this group of swimmers. Higher velocities do not allow measuring any force during some parts of the path since swimmers' force would be barely enough to overcome the water flow.

All the participants were familiar with tethered swimming. Additionally, they underwent a familiarization protocol with all the procedures. A belt was attached to the hip with a 2-m steel cable. Force recordings were synchronized with 3 different video cameras, using a video switcher (Roland Corporation, Roland Pro A/V V-1HD, Osaka, Japan). A visual-auditory signal was used to determine the start and the end of the 30-s. Before that, the participants swam for 5-s at low intensity, in order to avoid inertial effect, adapted from Barbosa ${ }^{21}$. To avoid interferences in force parameters caused by breathing, a snorkel was used for tethered swimming. Feet were restrained on a rope (figure 1). Placing the feet on the support allows swimmers to rotate and keep the horizontal position as if they were kicking. Moreover, both interaction with the arms and interfering with the measurements were avoided ${ }^{4}$.There were 15 minutes of active rest between each trial. After the trial, the participants were all asked for their rate of perceived exertion (RPE) ${ }^{22}$.

Forces were measured using a load-cell (HBM, RSCC S-Type, Darmstadt, Germany). The load cell was aligned with the direction of the swimming, recording at 200-Hz. Analog data were converted (Remberg, Force Isoflex, celula 1.4, Spain), registered and exported (National instruments, NI USB 600, Austin, USA) to a specific runtime environment developed using LabVIEW (National instruments, Austin, USA), allowing to visualize the recordings in real time. Stroke rate was recorded and analysed using Automatic Swimming Performance Analysis (A.S.P.A, project reference IE_57161), it allowed the collection 
of the performance data automatically from video frames. Technical details are provided elsewhere ${ }^{23}$.

\section{(Insert figure 1 near here)}

Swimming performance was measured using 3 distances; 25, 50 and $100-\mathrm{m}$ front crawl. An in-water start was used. During the 25-m a speedometer cable (lineal transducer, Heidenhain, D83301, Traunreu, Germany) was attached to the swimmer's hip by way of a belt, recording at $200-\mathrm{Hz}$. Data were recorded, converted (Signal Frame MF020, Sportmetrics, Spain) and exported to the software (Signalframe an v.2.00). Total time and stroke rate were recorded using A.S.P.A.

Force-time and velocity-time curves were smoothed using a fourth order Butterworth low pass digital filter, with a cut off frequency of $10 \mathrm{~Hz}$. The following parameters were estimated for each tethered swimming trial (Figure 2) ${ }^{5}$ :

- Maximum force (Fmax): highest value obtained from the individual force-time curve.

- Average force (Favg): mean of force values recorded during the 30 seconds.

- Maximum impulse (Imax): highest value of the impulse of force (equation 1) in a single stroke.

- Average impulse (Iavg): quotient of the sum of the singlestroke impulse and the number of strokes performed during the 30 -s tethered swim.

(Insert figure 2 near here)

Both velocity-time and force-time curves were examined, and 5 successive strokes were chosen for further analysis, adapted from Morouço ${ }^{17}$. The selected strokes occurred during midtesting. $d v$ and $d F$ were analysed as previously described ${ }^{17}$ :

$$
d v=\frac{\sqrt{\frac{\sum_{i}\left(v_{i}-v\right)^{2} \cdot A F_{i}}{n}}}{\frac{\sum_{i} v_{i} \cdot A F_{i}}{n}} \cdot 100
$$

Where $d v$ represents the intra-cyclic variation of the horizontal velocity of the hip, $v$ represents the mean swimming velocity, $v_{i}$ represents the instantaneous swimming velocity, $\mathrm{A} F_{i}$ represents the acquisition frequency, and $n$ is the number of measured strokes. To calculate $\mathrm{dF}$, the same equation was adapted using the force parameters obtained in the tethered swimming test, instead of the velocity parameters.

Swimmers indicated the RPE after each trial, using the adapted Borg's scale with incremental descriptors of the perception of exertion, ranging from 1 (no exertion at all) to 10 (maximal exertion $)^{22}$. 


\section{Statistical analysis}

The normality of all distributions was verified using ShapiroWilk test and visual inspection of histograms. For analytical purposes, Napierian logarithm was calculated. Parametric statistical analysis was adopted. Repeated measures ANOVA was performed to determine the differences between tethered swimming variables in the 4 conditions. It was also performed to determine the differences between swimming velocity, SR and RPE in 25, 50 and 100-m front crawl. Bivariate Pearson's correlation coefficients (r) were determined between selected variables, and simple linear regression analyses were applied to evaluate the potential associations.

Paired-sample t-test was used to assess differences, in SR and RPE, between $25-\mathrm{m}$ and tethered swimming at zero velocity. The same procedure was performed to compare SR and RPE between each free swimming distance and every tethered swimming condition.

The effect sizes (d) of the obtained differences were calculated and categorized (small if $0 \leq|\mathrm{d}| \leq 0.5$, medium if $0.5<|\mathrm{d}| \leq 0.8$, and large if $|\mathrm{d}|>0.8)^{24}$. All statistical procedures were performed using SPSS 23.0 (Chicago, IL, USA) and the level of statistical significance was set at $\mathrm{p}<0.05$.

\section{Results}

The mean \pm SD values for the tethered forces, grouped into water flow and swimming performance variables respectively are presented in tables 1 and 2. Repeated measures ANOVA analysis revealed significant differences for average force $\left(\mathrm{F}_{3,13}=207.318, \mathrm{p}<0.001\right)$, maximum force $\left(\mathrm{F}_{3,13}=73.631\right.$, $\mathrm{p}<0.001)$, average impulse $\left(\mathrm{F}_{3,13}=101.122, \mathrm{p}<0.001\right)$, maximum impulse $\left(\mathrm{F}_{3,13}=97.713, \mathrm{p}<0.001\right)$ and $\mathrm{dF}\left(\mathrm{F}_{3,13}=14.169, \mathrm{p}<0.001\right)$, between the 4 tethered swimming conditions. There were also significant differences for swimming velocities $\left(\mathrm{F}_{2,14}=211.471\right.$, $\mathrm{p}<0.001)$, between the 3 distances. Stroke rate was not significantly different between tethered swimming in the 4 conditions $\left(\mathrm{F}_{3,13}=0.076, \mathrm{p}=0.972\right)$ yet it was significantly different between 25, 50 and 100-m $\left(\mathrm{F}_{2,14}=25.311, \mathrm{p}<0.001\right)$. Likewise, RPE was significantly different between 25,50 and $100-\mathrm{m}\left(\mathrm{F}_{2,14}=44.596, \mathrm{p}<0.001\right)$, but it was not significantly different between the 4 conditions of tethered swimming $\left(\mathrm{F}_{3,13}=2.402, \mathrm{p}=0.115\right)$. Post-hoc analysis showed that tethered forces were higher at lower velocities $(p<0.001)$, except $d F$, which was higher as the velocity increased $(p<0.001)$. Mean velocity in $25-\mathrm{m}$ was higher than mean velocity in 50-m and 100$\mathrm{m}(\mathrm{p}<0.001)$. SR was higher in the $25-\mathrm{m}(\mathrm{p}<0.001)$ and RPE was higher in the $100-\mathrm{m}(\mathrm{p}<0.001)$. 
(Insert Table 1 near here)

\section{(Insert Table 2 near here)}

Table 3 shows Pearson's correlations of tethered swimming variables at different water flow velocities and free swimming performance. Simple linear regression analysis shows positive associations of velocity in 25-m with all tethered force variables at $1.329 \mathrm{~m} \cdot \mathrm{s}^{-1}$ water flow velocity (Figure 3 ). Maximum force was positively associated with velocity in $50-\mathrm{m}(\mathrm{r}=0.52$; $\mathrm{p}=0.39$ ). Average force, maximum force and maximum impulse at $1.124 \mathrm{~m} \cdot \mathrm{s}^{-1}$ water flow velocity were positively associated with velocity in $25-\mathrm{m}(\mathrm{r}=0.565, \mathrm{r}=0.523$ and $\mathrm{r}=0.627 ; \mathrm{p}=0.023$, $\mathrm{p}=0.038$ and $\mathrm{p}=0.009$ respectively). There were associations between $\mathrm{dF}$, at zero velocity and $1.389 \mathrm{~m} \cdot \mathrm{s}^{-1}$ water flow velocity, and $\mathrm{dv} \quad(\mathrm{r}=0.507$ and $\mathrm{r}=0,436 ; \mathrm{p}=0.022$ and $\mathrm{p}=0.045$ respectively). However, there was no significant association between $\mathrm{dF}$ and $\mathrm{dv}$ with swimming performance.

(Insert Table 3 near here)

(Insert Figure 3 near here)

Results showed significant differences in SR and RPE between tethered swimming in the 4 conditions and 25 , and $100-\mathrm{m}$ $(p<0.05)$, yet no significant differences between SR and RPE in $50-\mathrm{m}$ and tethered swimming in the 4 conditions

\section{Discussion:}

The main finding of this study was that tethered swimming variables measured at different water flow velocities were positively associated to 25 and $50-\mathrm{m}$ swimming velocities. Our results confirm the established hypothesis; the association is higher when the flume velocity approaches the free-swimming velocity.

With free-swimming velocity increasing the force production declines; diminishing the capability to apply force ${ }^{1}$. At zero velocity this is unnoticeable as there is no displacement, while including the water flow simulates the displacement in the water ${ }^{19}$. Surprisingly, swimmers with lower level of force at zero velocity were able to develop higher values at high water flow velocities than their stronger teammates, being also the faster swimmers ${ }^{19}$. Thus, including the water flow in tethered swimming seems to evaluate the ability of the swimmers to effectively apply force in the water while tethered swimming at zero velocity seems to measure the muscle strength potential of the swimmer. This fact explains why the relationship between tethered swimming and swimming performance becomes stronger when the water flow increases. This is of crucial 
importance, as performance depends on the ability to effectively apply force in the water, rather than on the relative force of the swimmers 4 .

Relationships have been shown when comparing pulling force at zero velocity and 8 different water flow velocities with 100 -m swimming velocity ${ }^{19}$. Former authors compared elite swimmers using 100-m competitive mean swimming velocity in front crawl. This might explain why our results did not show an association between tethered swimming variables and 100-m. The first point to consider is that, we used swimming velocity measured in short course, where turning might affect the outcome ${ }^{25}$. Secondly, $100-\mathrm{m}$ is a distance with a different contribution from the aerobic and anaerobic systems compared to 25 or $50-\mathrm{m}^{26}$. Thus, swimmers aerobic and anaerobic capacity plays an important role. Thirdly, the heterogeneity in the sample level might have affected this relationship. Besides, the magnitude of the main forces identified in this study was considerably lower than previously presented ${ }^{19}$. However, there is an important difference in test time (30-s versus 5-s). This fact added to the restriction of the legs might explain the considerable difference in the forces obtained.

The force produced when swimming has been compared between tethered swimming and other experimental techniques. The mean propulsive force obtained using the Assisted Towing Method is not closely related to tethered swimming at zero velocity ${ }^{7}$. However, tethered swimming at zero velocity measured the muscle strength potential of the swimmers ${ }^{6,8,9}$, not the ability to effectively apply force in the water. Therefore, the fact that tethered swimming in a flume is a more similar situation to assisted towing method than at zero velocity, might increase the association of force obtained by these 2 different methods. More research is required to better understand this association. Comparing our results at zero velocity with previous studies it is unclear which is the best tethered variable to be assessed. Average force was a reliable parameter to estimate swimming velocity ${ }^{27}$. Conversely, maximum impulse showed a better association with performance. This difference might be explained by the swimmers' level. Elite sprint swimmers can take advantage at each phase of the stroke, relying more on their stroke frequency to increase the very high swimming velocity developed. Thus, impulse should always be taken into consideration in top swimmers ${ }^{15}$. The magnitude of Fmax, Favg, Imax, and Iavg identified in this study at zero velocity is in line with those found in previous studies with the same test duration and conditions ${ }^{5}$.

The $\mathrm{dF}$ was directly related to the water flow, becoming higher as the water flow increased. The levels of forces were lower during both the propulsive and non-propulsive moments as the water flow velocity increased. Therefore, the restriction of the legs might have affected the association of our results with 
swimming performance. Regarding $\mathrm{dv}$, the no association presented in this study and the different results obtained previously ${ }^{17,28}$ demonstrate that more research is required to better understand this relationship. Nevertheless, it seems that $\mathrm{dF}$ is better related to performance than $\mathrm{dv}$

Stroke Rate and RPE were not significantly different between the 30-s tethered swimming (all conditions) and 50-m free swimming. These results confirm that 30 -s tethered swimming replicate the effort of 50-m free swimming ${ }^{5}$. Equally, results showed significant differences between the 30-s tethered swimming in the 4 conditions and 25 and $100-\mathrm{m}$ free swimming. Thus, we can assume that 30 -s tethered swimming is not able to replicate the effort over those given distances. Conversely, 15 or 60 -s in tethered swimming may replicate the effort of a 25 and $100-\mathrm{m}$ respectively, since it is approximately the time needed to cover those distances ${ }^{29}$.

The fact that the association between arm stroke tethered was studied with swimming front crawl free swimming and not with arm stroke free swimming was a point of discussion. However, the restriction of the legs during swimming could have affected the results, if swimmers had had to wear a pull-boy or a band on their ankle, the effect on each swimmer would have been different, thus making it impossible to control its effects. This fact, added to the high contribution of arms during front crawl sprint ${ }^{30}$ was determinant to not restricting the legs action during free swimming.

This is the first study investigating the association between tethered variables at zero, $0.926,1.124$ and $1.389 \mathrm{~m} \cdot \mathrm{s}^{-1}$ water flow velocities and 25, 50 and $100-\mathrm{m}$ swimming velocities, obtaining higher association between force variables and 25 and 50 -m performance at higher water flow velocities.

\section{Practical applications}

Our results will help coaches to evaluate their swimmers' ability to effectively apply force in the water. Comparing their results during the whole season might determine if performance improvements are due to enhancement on the ability to apply force in the water. Future research might study whether tethered swimming variables at high water flow velocities are affected by strength training. Thus, coaches would be able to know if strength gains are transferred in swimming performance improvements. Moreover, the fact that tethered swimming in a flume and free swimming are similar situations facilitates physiological measurements such as $\mathrm{VO}_{2 \max }$, relating it to force measurements. Future research should examine if there are kinematical differences between tethered swimming in a flume and free swimming. This would allow more complete biomechanical analyses and to compare how technical changes affect the force applied by the swimmers. 


\section{Conclusion}

The relevance of our study is that by using a swimming flume, tethered swimming becomes a similar situation to free swimming. It allows to measure the ability of the swimmers to effectively apply force in the water, obtaining a more accurate relationship, between all tethered swimming force variables and swimming performance in 25 and $50-\mathrm{m}$. The relationship is stronger as the water flow velocity increases and approaches the actual free-swimming velocity. Measuring at zero velocity position may underestimate the relationships between force variables and swimming performance since it measures the strength potential of the swimmers. Our results do not clarify the controversy of using intra-cyclic velocity variation and intracyclic force variation. Finally, it is important to mention that the similarities shown between tethered swimming and free swimming in stroke rate and RPE, enhance the use of tethered swimming in a flume as a proper tool for assessing and training.

\section{Acknowledgments}

This study was supported by grant awarded by the Ministry of Economy, Industry and Competitiveness (Spanish Agency of Research) and the European Regional Development Fund (ERDF); DEP2014-59707-P “SWIM: Specific Water Innovative Measurements applied to the development of International Swimmers in Short Swimming Events (50 and $100 \mathrm{~m})$ and the Spanish Ministry of Education, Culture and Sport: FPU17/02761 grant. This article is a part of an international thesis belonging to the Program of $\mathrm{PhD}$ in Biomedicine (B11.56.1), from the University of Granada, Granada (Spain).

\section{References:}

1. Keskinen KL, Tilli LJ, Komi PV. Maximum velocity swimming: Interrelationships of stroking characteristics, force production and anthropometric variables. Scand $J$ Sport sci. 1989;11:87-92.

2. Barbosa TM, Bragada JA, Reis VM, Marinho DA, Carvalho C, Silva AJ. Energetics and biomechanics as determining factors of swimming performance: Updating the state of the art. $J$ Sci Med Sport. 2010;13(2):262-269. doi:10.1016/j.jsams.2009.01.003

3. Narita K, Nakashima M, Takagi H. Developing a methodology for estimating the drag in front-crawl swimming at various velocities. $J$ Biomech. 2017;54:123-128. doi:10.1016/j.jbiomech.2017.01.037

4. Dominguez-Castells R, Izquierdo M, Arellano R. An updated protocol to assess arm swimming power in front crawl. Int J Sports Med. 2013;34(4):324-329. 
doi:10.1055/s-0032-1323721

5. Morouço PG, Marinho DA, Keskinen KL, Badillo JJ, Marques MC. Tethered swimming can be used to evaluate force contribution for short-distance swimming performance. J Strength Cond Res. 2014;28(11):30933099.

6. Akis T, Orcan Y. Experimental and analytical investigation of the mechanics of crawl stroke swimming. Mech Res Commun. 2004;31(2):243-261. doi:10.1016/j.mechrescom.2003.07.001

7. Mason B, Formosa D, Rollason S. A Comparison Between the Values Obtained From Active Drag Analysis Compared To Forces Produced in Tethered Swimming. ISBS-Conference Proc Arch. 2009;1(1). https://ojs.ub.uni-konstanz.de/cpa/article/view/3140.

8. Amaro N, Marinho DA, Batalha N, Marques MC, Morouço P. Reliability of tethered swimming evaluation in age group swimmers. J Hum Kinet. 2014;41:155-162. doi:10.2478/hukin-2014-0043

9. Kjendlie PL, Thorsvald K. A tethered swimming power test is highly reliable. Port J Sport Sci. 2006;6(2):231233.

10. Maglischo CW, Maglischo EW, Sharp RL, Zier DJ, Katz A. Tethered and nontethered crawl swimming. In: Proceedings of the ISBS: Sports Biomechanics.; 1984:163-176.

11. Samson M, Monnet T, Bernard A, Lacouture P, David L. Comparative study between fully tethered and free swimming at different paces of swimming in front crawl. Sport Biomech. 2018:1-16. doi:10.1080/14763141.2018.1443492

12. Amaro NM, Morouço PG, Marques MC, Fernandes RJ, Marinho DA. Biomechanical and bioenergetical evaluation of swimmers using fully-tethered swimming: A qualitative review. J Hum Sport Exerc. 2017;12(4):1346-1360. doi:10.14198/jhse.2017.124.20

13. Morouço P, Keskinen KL, Vilas-Boas JP, Fernandes RJ. Relationship between tethered forces and the four swimming techniques performance. $J$ Appl Biomech. 2011;27(2):161-169. doi:10.1123/jab.27.2.161

14. Neiva H, Marques MC, Marinho DA, et al. The Effect of Warm-up on Tethered Front Crawl Swimming Forces. $J$ Hum Kinet. 2011;(Special Issue):113-119. doi:10.2478/v10078-011-0066-1

15. Dopsaj M, Matkovíc I, Zdravkovíc I. The relationship between 50-m Freestyle results and characteristics of tethered forces in male sprinters: A new approach to tethered swimming test. Phys Educ Sport. 2000;1:15-22.

16. Knudson D V. Correcting the use of the term "power" in the strength and conditioning literature. $J$ strength Cond 
Res. 2009;23(6):1902-1908.

http://content.wkhealth.com/linkback/openurl?sid=WKP TLP:landingpage\&an=00124278-20090900000038\%5Cnhttp://www.ncbi.nlm.nih.gov/pubmed/19675 467.

17. Morouço PG, Barbosa T, Arellano R, Vilas-Boas JP. Intra-Cyclic Variation of Force and Swimming Performance. Int J Sports Physiol Perform. 2017;0(0):120. doi:10.1123/ijspp.2017-0223

18. Vilas-Boas JP, Fernandes RJ, Barbosa TM. Intra-cycle velocity variations, swimming economy, performance and training in swimming. In: The World Book Of Swimming: From Science To Performance. New York: Nova Science Publishers; 2011.

19. Vorontsov A, Popov O, Binevsky D, Dyrko V. The assessment of specific strength in well trained male athletes during tethered swimming in the swimming flume. Rev Port Ciências do Desporto. 2006:275-277.

20. McLean SP, Palmer D, Ice G, Truijens M, Smith JC. Oxygen uptake response to stroke rate manipulation in freestyle swimming. Med Sci Sports Exerc. 2010;42(10):1909-1913. doi:10.1249/MSS.0b013e3181d9ee87

21. Barbosa AC, de Souza Castro F, Dopsaj M, Cunha SA, Júnior OA. Acute responses of biomechanical parameters to different sizes of hand paddles in frontcrawl stroke. J Sports Sci. 2013;31(9):1015-1023. doi:10.1080/02640414.2012.762597

22. Borg GA. Psychophysical bases of perceived exertion. Med sci Sport Exerc. 1982;14(5):377-381. doi:10.1249/00005768-198205000-00012

23. Arellano R, Ruiz-Teba A, Morales E, Gay A, CuencaFernández F, López-Contreras G. Short course 50m female freestyle performance comparison between national and regional swimmers. In: XIIIth International Symposium on Biomechanics and Medicine in Swimming. ; 2018:348-355.

24. Cohen J. Statistical power analysis for the behavioural sciences. In: Hillsdale, NJ: Lawrence Erlbaum Associates; 1988:20-27.

25. Veiga S, Roig A, Gómez-Ruano MA. Do faster swimmers spend longer underwater than slower swimmers at World Championships? Eur J Sport Sci. 2016;16(8):919-926. doi:10.1080/17461391.2016.1153727

26. Maglischo EW. Swimming Fastest. Human Kinetics; 2003.

27. Taylor S, Lees A, Stratton G, Maclaren D. Reliability of force production in tethered freestyle swimming among competitive age-group swimmers. J Sport Sci. 
2001;19:12-13.

28. Barbosa TM, Lima F, Portela A, et al. Relationships between energy cost, swimming velocity and speed fluctuation in competitive swimming strokes. Port $J$ Sport Sci. 2006;6(2):192-194.

29. Kalva-Filho CA, Zagatto AM, Araújo MIC, et al. Relationship Between Aerobic and Anaerobic Parameters From 3-Minute All-Out Tethered Swimming and 400-m Maximal Front Crawl Effort. $J$ Strength Cond Res. 2015;29(1):238-245.

30. Morouço PG, Marinho DA, Izquierdo M, Neiva H, Marques MC. Relative Contribution of Arms and Legs in $30 \mathrm{~s}$ Fully Tethered Front Crawl Swimming. Biomed Res Int. 2015;2015. doi:10.1155/2015/563206 


\section{Figure captions and tables}

Figure 1. Swimmers' real situation during tethered swimming in the flume.

Figure 2. Example of 3 consecutive stroke cycles front crawl force recordings. The main analysis points are shown. Each curve corresponds to each arm. Fmax: maximum force; Fmin: minimum force; IMP: impulse.

Figure 2. Linear regressions between tethered force variables at $1.389 \mathrm{~m} \cdot \mathrm{s}^{-1}$ water flow velocity and velocity in $25-\mathrm{m}(\mathrm{p}<0,05)$. Individual value and $95 \%$ confidence lines are represented. A) AVER FORCE: Average force; B) MAX FORCE: maximum force; C) AVER IMP: average impulse; D) MAX IMP: maximum impulse; V25m: velocity in 25 .

Table 1. Mean \pm SD values for the tethered swimming variables, rate of perceived exertion and stroke rate, grouped by water flow velocity

Table 2. Mean \pm SD values for swimming performance variables and rate of perceived exertion

Table 3. Pearson's correlation of tethered swimming variables at different water flow velocities with swimming performance 


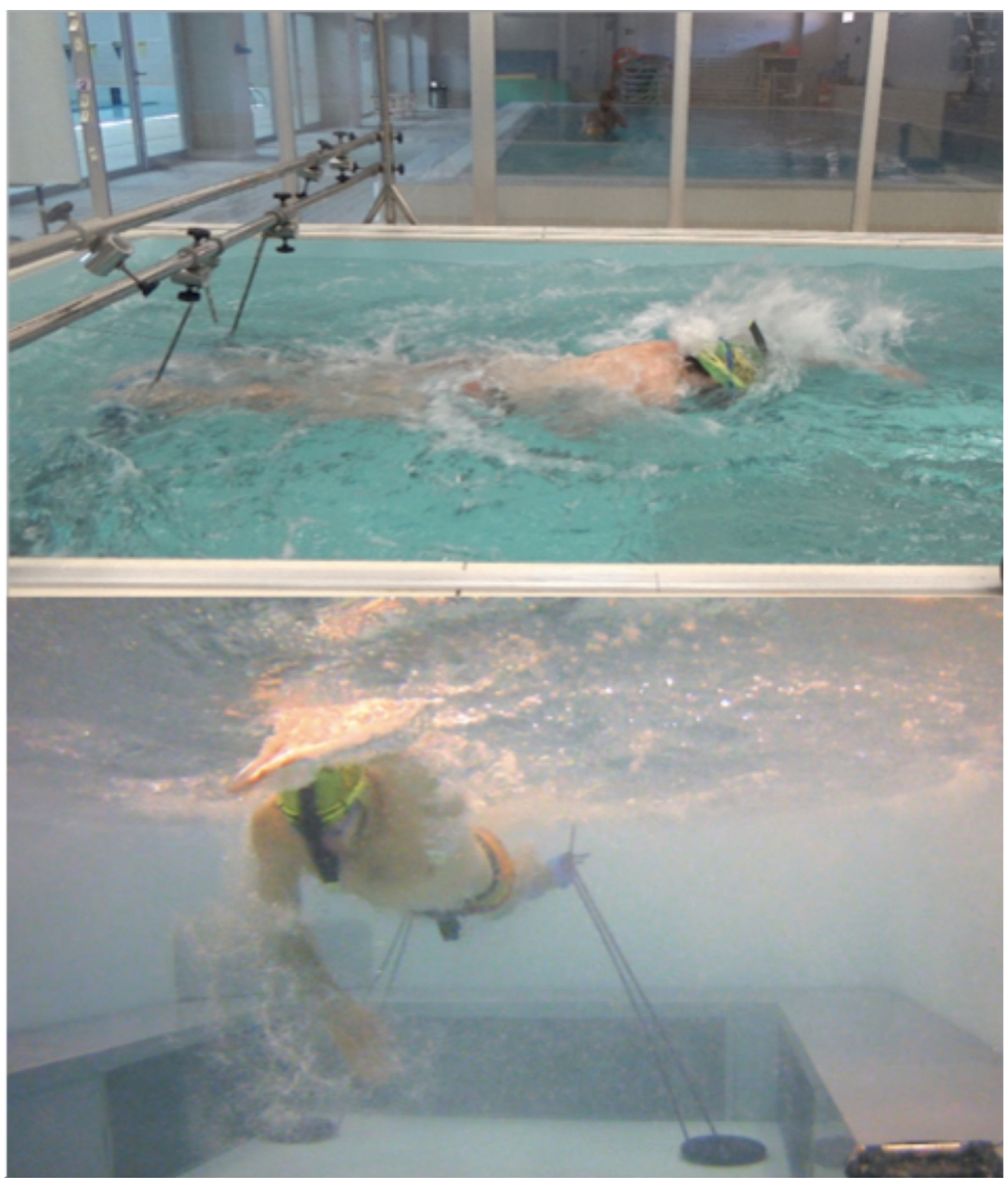

Figure 1. Swimmers' real situation during tethered swimming in the flume.

$99 \times 117 \mathrm{~mm}(300 \times 300 \mathrm{DPI})$ 


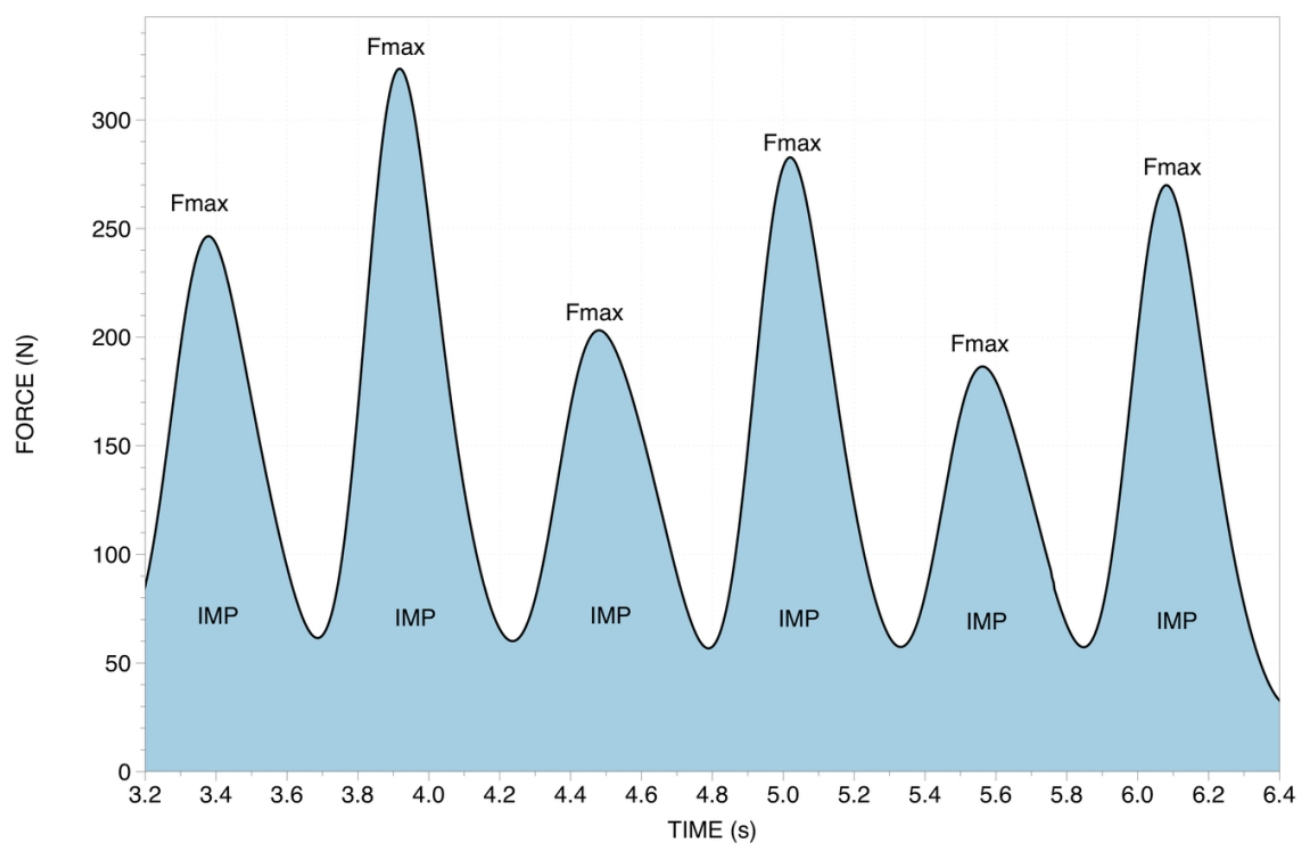

Figure 2. Example of 3 consecutive stroke cycles front crawl force recordings. The main analysis points are shown. Each curve corresponds to each arm. Fmax: maximum force; Fmin: minimum force; IMP: impulse.

$114 \times 74 \mathrm{~mm}(300 \times 300 \mathrm{DPI})$ 
A)

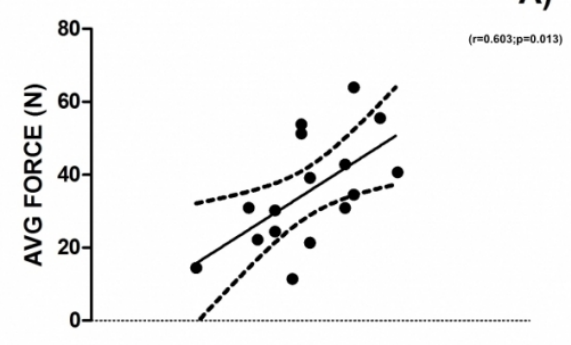

C)

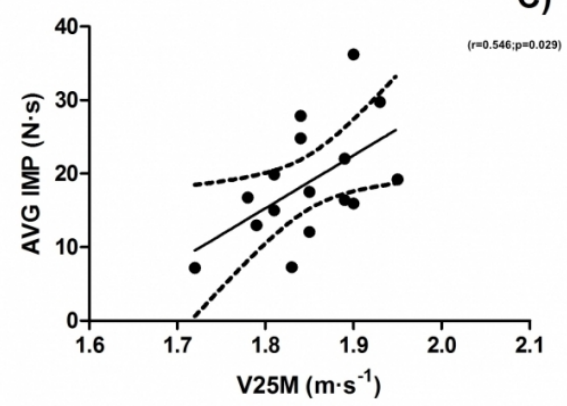

B)

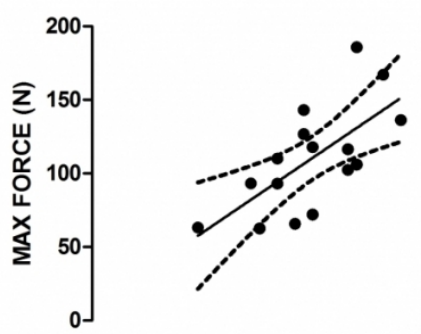

D)

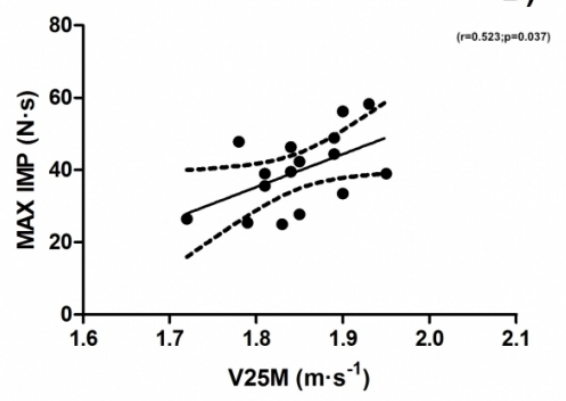

Figure 2. Linear regressions between tethered force variables at $1.389 \mathrm{~m} \square \mathrm{s}-1$ water flow velocity and velocity in $25-m(p<0,05)$. Individual value and $95 \%$ confidence lines are represented. A) AVER FORCE: Average force; B) MAX FORCE: maximum force; C) AVER IMP: average impulse; D) MAX IMP: maximum impulse; V25m: velocity in 25.

$476 \times 310 \mathrm{~mm}(72 \times 72 \mathrm{DPI})$ 
Table 1. Mean \pm SD values for the tethered swimming variables, rate of perceived exertion and stroke rate, grouped by water flow velocity

\begin{tabular}{ccccc}
\hline & $\begin{array}{c}\text { Water flow velocity: } \\
\mathbf{0 ~} \mathbf{~ m} \cdot \mathbf{s}^{-1}\end{array}$ & $\begin{array}{c}\text { Water flow velocity: } \\
\mathbf{0 . 9 2 6} \mathbf{~ m} \cdot \mathbf{s}^{-1}\end{array}$ & $\begin{array}{c}\text { Water flow velocity: } \\
\mathbf{1 . 1 2 4} \mathbf{~ m} \cdot \mathbf{s}^{-1}\end{array}$ & $\begin{array}{c}\text { Water flow velocity: } \\
\mathbf{1 . 3 8 9} \mathbf{~ m} \cdot \mathbf{s}^{-1}\end{array}$ \\
\hline Favg (N) & $93.20 \pm 16.92$ & $60.14 \pm 18.23$ & $43.89 \pm 15.32$ & $35.49 \pm 15.23$ \\
Fmax (N) & $214.58 \pm 48.66$ & $156.55 \pm 37.00$ & $125.14 \pm 38.86$ & $110.11 \pm 36.18$ \\
Iavg (N·s) & $50.16 \pm 10.92$ & $31.97 \pm 8.76$ & $23.56 \pm 8.23$ & $18.80 \pm 7.89$ \\
Imax (N·s) & $78.75 \pm 13.70$ & $58.83 \pm 13.65$ & $47.28 \pm 11.21$ & $39.74 \pm 10.44$ \\
dF (\%) & $39.72 \pm 8.15$ & $47.58 \pm 10.64$ & $50.07 \pm 13.65$ & $53.56 \pm 11.72$ \\
RPE & $8.25 \pm 1.06$ & $8.13 \pm 0.95$ & $8.56 \pm 0.72$ & $8.56 \pm 0.96$ \\
SR (Hz) & $0.92 \pm 0.10$ & $0.92 \pm 0.08$ & $0.92 \pm 0.08$ & $0.92 \pm 0.10$ \\
\hline
\end{tabular}

Abbreviations: Favg, average force; Fmax, maximum force; Iavg, average impulse; Imax, maximum impulse; $\mathrm{dF}$, intra-cyclic force variation; RPE, rate of perceived exertion; SR, stroke rate 
Table 2. Mean \pm SD values for swimming performance variables and rate of perceived exertion

\begin{tabular}{cccc}
\hline & $\mathbf{2 5}-\mathbf{m}$ & $\mathbf{5 0 -}-\mathbf{m}$ & $\mathbf{1 0 0}-\mathbf{m}$ \\
\hline $\mathrm{SV}\left(\mathrm{m} \cdot \mathrm{s}^{-1}\right)$ & $1.84 \pm 0.05$ & $1.80 \pm 0.06$ & $1.66 \pm 0.06$ \\
$\mathrm{RPE}$ & $7.38 \pm 0.80$ & $8.69 \pm 0.60$ & $9.44 \pm 0.62$ \\
$\mathrm{SR}(\mathrm{Hz})$ & $1.01 \pm 0.13$ & $0.92 \pm 0.9$ & $0.81 \pm 0.05$ \\
$\mathrm{dv}(\%)$ & $8.08 \pm 1.82^{*}$ & & \\
\hline
\end{tabular}

Abbreviations: SV, swimming velocity; RPE, rate of perceived exertion; SR, stroke rate; dv, intra-cyclic velocity variation. * Speedometer additional data. 
1 Table 3. Pearson's correlation of tethered swimming variables at different water flow velocities with swimming performance

\begin{tabular}{|c|c|c|c|c|c|c|c|c|c|c|c|c|c|c|c|c|c|c|c|c|c|}
\hline & \multicolumn{5}{|c|}{ Water flow velocity: $0 \mathrm{~m} \cdot \mathrm{s}^{-1}$} & \multicolumn{5}{|c|}{ Water flow velocity: $0.926 \mathrm{~m} \cdot \mathrm{s}^{-1}$} & \multicolumn{5}{|c|}{ Water flow velocity: $1.124 \mathrm{~m} \cdot \mathrm{s}^{-1}$} & \multicolumn{5}{|c|}{ Water flow velocity: $1.389 \mathrm{~m} \cdot \mathrm{s}^{-1}$} & \multirow[b]{2}{*}{$\mathrm{dv}$} \\
\hline & Favg & Fmax & Iavg & $\operatorname{Imax}$ & $\mathrm{dF}$ & Favg & Fmax & Iavg & $\operatorname{Imax}$ & $\mathrm{dF}$ & Favg & Fmax & Iavg & $\operatorname{Imax}$ & $\mathrm{dF}$ & Favg & Fmax & Iavg & $\operatorname{Imax}$ & $\mathrm{dF}$ & \\
\hline $\begin{array}{c}\mathrm{SV} \\
25-\mathrm{m}\end{array}$ & $0.435^{*}$ & 0.271 & 0.196 & $0.455^{*}$ & $0 . \overline{299}$ & $0.436^{*}$ & 0.414 & $0.439^{*}$ & $0.445^{*}$ & 0. & $0.565^{*}$ & $0.523^{*}$ & $0.483^{*}$ & $0.627^{* *}$ & $0 . \overline{292}$ & $0.603^{* *}$ & $0.673^{* *}$ & $0.546^{*}$ & $0.523^{*}$ & 0.033 & 0.101 \\
\hline $\begin{array}{c}\mathrm{SV} \\
50-\mathrm{m}\end{array}$ & 0.268 & 0.138 & 0.083 & 0.380 & $\begin{array}{c}- \\
0.290\end{array}$ & 0.222 & 0.244 & 0.229 & 0.291 & $\begin{array}{c}- \\
0.133\end{array}$ & 0.415 & 0.418 & 0.359 & $0.472^{*}$ & $\begin{array}{c}- \\
0.319\end{array}$ & $0.476^{*}$ & $0.520^{*}$ & $0.465^{*}$ & 0.424 & $0 . \overline{213}$ & $0 . \overline{112}$ \\
\hline $\begin{array}{c}\mathrm{SV} \\
100-\mathrm{m}\end{array}$ & 0.351 & 0.187 & 0.172 & $0.442^{*}$ & $\overline{-}-$ & 0.263 & 0.228 & 0.302 & 0.298 & $0 . \overline{248}$ & 0.358 & 0.357 & 0.322 & $0.494^{*}$ & $\begin{array}{c}- \\
0.376\end{array}$ & 0.396 & $0.435^{*}$ & 0.415 & 0.405 & $0 . \overline{238}$ & $\begin{array}{c}- \\
0.028\end{array}$ \\
\hline
\end{tabular}

2 Abbreviations: Favg, average force; Fmax, maximum force; Iavg, average impulse; Imax, maximum impulse; dF, intra-cyclic force variation; dv, intra-cyclic velocity variation; SV25-m, swimming velocity in $25 \mathrm{~m}$ front crawl; SV50-m, swimming velocity in 50-m front crawl; SV100-m, swimming velocity in $100-\mathrm{m}$ front crawl. ${ }^{*} \mathrm{p}<0,05 . * * \mathrm{p}<0,01$. 